\title{
(6) OPEN ACCESS \\ Increasing the dose of television advertising in a national antismoking media campaign: results from a randomised field trial
}

\author{
Tim McAfee, ${ }^{1}$ Kevin C Davis, ${ }^{2}$ Paul Shafer, ${ }^{2}$ Deesha Patel, ${ }^{1}$ Robert Alexander, ${ }^{1}$ \\ Rebecca Bunnell ${ }^{1}$
}

${ }^{1}$ Office on Smoking and Health, National Center for Chronic Disease Prevention and Health Promotion, Centers for Disease Control and Prevention, Atlanta, Georgia, USA

${ }^{2}$ Center for Health Policy Science and Tobacco Research RTI International, Research Triangle Park, North Carolina, USA

\section{Correspondence to} Dr Tim McAfee, Office on Smoking and Health, Centers for Disease Control and Prevention, 4770 Buford Hwy, MS-K50, Atlanta, GA 30341, USA; mtt4@cdc.gov

Received 29 June 2015 Accepted 30 October 2015 Published Online First 17 December 2015

\section{(1) corssank}

To cite: McAfee $T$ Davis KC, Shafer $\mathrm{P}$, et al. Tob Control 2017;26:1928.

\section{ABSTRACT \\ Background While antismoking media campaigns} have demonstrated effectiveness, less is known about the country-level effects of increased media dosing. The 2012 US Tips From Former Smokers (Tips) campaign generated approximately 1.6 million quit attempts overall; however, the specific dose-response from the campaign was only assessed by self-report.

Objective Assess the impact of higher ad exposure during the 2013 Tips campaign on quit-related behaviours and intentions, campaign awareness, communication about campaign, and disease knowledge.

Methods A 3-month national media buy was supplemented within 67 (of 190) randomly selected local media markets. Higher-dose markets received media buys 3 times that of standard-dose markets. We compared outcomes of interest using data collected via web-based surveys from nationally representative, address-based probability samples of 5733 cigarette smokers and 2843 non-smokers.

Results In higher-dose markets, $87.2 \%$ of smokers and $83.9 \%$ of non-smokers recalled television campaign exposure versus $75.0 \%$ of smokers and $73.9 \%$ of nonsmokers in standard-dose markets. Among smokers overall, the relative quit attempt rate was $11 \%$ higher in higher-dose markets ( $38.8 \%$ vs $34.9 \% ; p<0.04)$. The higher-dose increase was larger in African-Americans $(50.9 \%$ vs $31.8 \% ; p<0.01)$. Smokers in higher-dose markets without a mental health condition, with a chronic health condition, or with only some college education made quit attempts at a higher rate than those in standard-dose markets. Non-smokers in higherdose markets were more likely to talk with family or friends about smoking dangers ( $43.1 \%$ vs $35.7 \%$; $\mathrm{p}<0.01$ ) and had greater knowledge of smoking-related diseases.

Conclusions The US 2013 Tips antismoking media campaign compared standard and higher doses by randomisation of local media markets. Results demonstrate the effectiveness of a higher dose for engaging non-smokers and further increasing quit attempts among smokers, especially African-Americans.

\section{INTRODUCTION}

Globally, one tobacco-related death occurs approximately every 6 seconds, totalling about five million deaths annually. ${ }^{1}$ Deaths in the USA account for nearly $10 \%$ of the global death toll. ${ }^{2}$ The WHO recommends that all countries implement MPOWER, ${ }^{3}$ a multicomponent comprehensive approach to tobacco control. The 'W' in
MPOWER stands for "Warn about the dangers of tobacco use', referring to public education campaigns. However, public education campaigns require significant financial commitments. Although the effectiveness of tobacco campaigns has been established, ${ }^{4-13}$ important questions remain about how to optimise investment of scarce tobacco education campaign resources.

In 2012, the US Centers for Disease Control and Prevention (CDC) launched Tips From Former Smokers (Tips), the first federally funded, national tobacco education campaign in the USA. An estimated 1.6 million cigarette smokers made a quit attempt, and more than 100000 likely quit smoking permanently because of the 3 -month campaign. ${ }^{8}$ The 2012 campaign also was associated with increased intentions to quit smoking and changes in beliefs about smoking-related risks. ${ }^{14}$ Building on this success, CDC launched the second campaign in 2013. The 2013 Tips campaign aired for 16 weeks (4 March-21 June) and featured similar creative content as the 2012 campaign: graphic, emotional advertisements featuring people telling their true stories of suffering from smoking-related diseases, including chronic obstructive pulmonary disease (COPD), smoking-related diabetes complications and respiratory effects from secondhand smoke. ${ }^{8} 15$ The 2013 campaign aired nationally, with a significant national media buy on cable television (TV) and complementary ads in radio, print (figure 1), billboard, transit and digital outlets. All video, radio and print ads can be found at http://www.cdc.gov/tips. ${ }^{15}$

The 2012 Tips campaign had a ubiquitous national buy and a local buy-up strategy that focused on high-prevalence localities; consequently, a randomised field trial with a non-exposed group to assess the effect of increased media exposure was not possible. Very few studies have been conducted that control the intensity of media buys with the intention of evaluating the impact of dose on outcomes. ${ }^{16}{ }^{17}$ For example, from 2000 to 2002, several matched pairs of US communities were randomised to either increased or standard doses of the national 'truth' campaign. ${ }^{16} \mathrm{~A}$ similar matchedpair design with random assignment to increased or standard media doses was conducted in 2007 among several rural communities as part of Legacy's 'truth or consequences' campaign evaluation. ${ }^{17}$ Both these studies generated modest to moderate contrasts in community-level exposure. However, they did not demonstrate, based on randomisation status, that these exposure contrasts were sufficient to influence changes in 


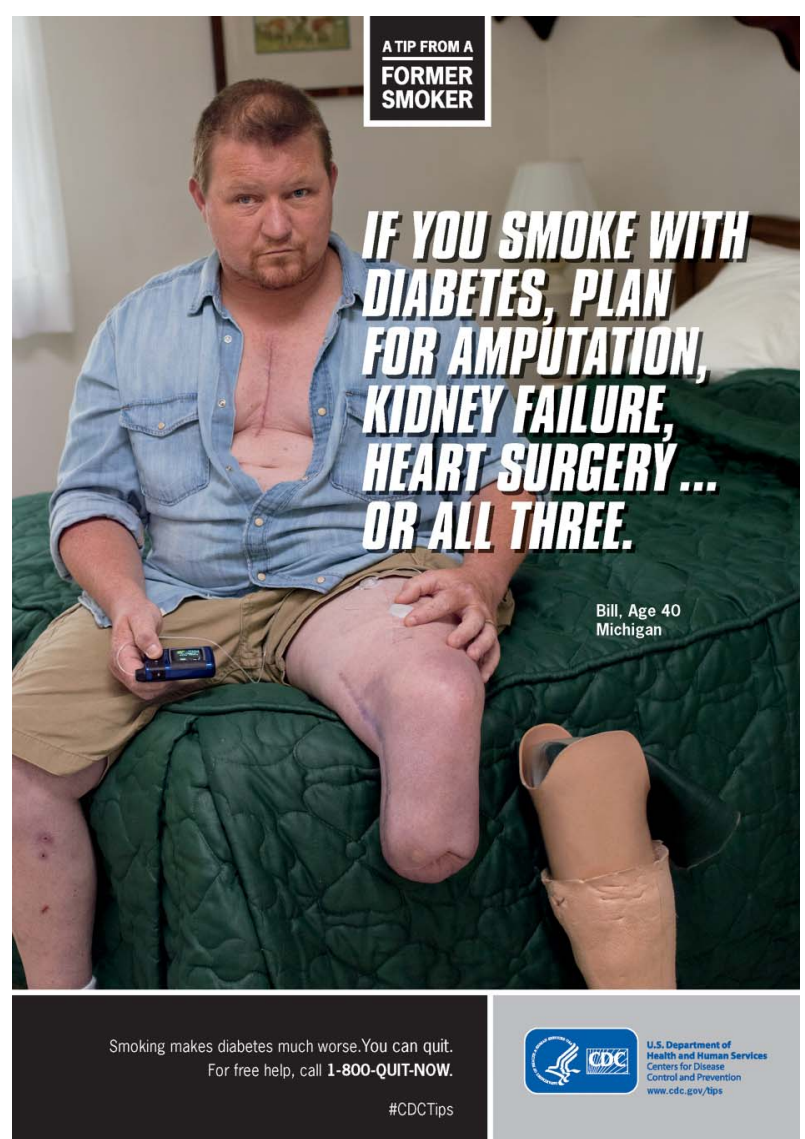

Figure 1 Print advertisement from the 2013 Tips campaign. CDC, Centers for Disease Control and Prevention; Tips, Tips From Former Smokers.

tobacco-related behavioural outcomes. Because even small changes in quit attempt rates are important at the population level, large samples are also necessary to detect effects.

As demonstrated by these studies, field-based designs with controlled media are difficult and expensive to implement, and they may not yield sufficient exposure contrast without prohibitively large evaluation sample sizes. For example, the 'truth' campaign study ${ }^{16}$ using matched pairs of communities with random media dosing assignments ultimately relied on selfreported ad exposure, without regard to randomisation category to detect outcome differences. Consequently, as part of the 2013 Tips evaluation, we sought to determine the impact of increasing the dose of ad exposure on smokers and nonsmokers, given previous evidence of standard-dose effectiveness. ${ }^{8}$ Furthermore, we wanted to determine the effect of a higher dose in priority subpopulations, including African-Americans, Hispanics, those with chronic mental and physical diseases, and those not completing college. Therefore, in addition to a standard-dose national media buy, 2013 Tips increased ad exposure in randomly selected local markets to assess the dose-response impact on campaign awareness, quit-related behaviours and intentions, communication about the campaign, and disease knowledge among smokers and non-smokers.

\section{METHODS}

\section{Study design}

The 2013 Tips campaign built on the research and approach used in the development of the 2012 Tips campaign ads. Real people (not actors) with smoking-related health conditions were featured in five new 30 second TV ads focusing on the impact of smoking on quality of life. The new ads were aired along with six ads from 2012, from 4 March 2013 to 17 June 2013. In addition, there was a much smaller ad component consisting of radio, billboards and print media featuring some of the same ad participants, as well as a website featuring ads, ad participants and cessation support materials. Doses of these media channels were not manipulated in the experiment (only TV dose was increased).

The 2013 campaign aimed to air an average of 800 TV gross rating points (GRPs) to all US markets. GRPs are a standard measure of advertising intensity calculated at the market level by Nielsen Media Research based on TV viewership estimates. Ad GRPs are defined as the product of the percentage of the population potentially exposed to advertising (reach) and the average number of times the ads were seen (frequency). GRPs have been used in multiple evaluations of tobacco counter-advertising campaigns. ${ }^{5} 1012 \quad 18$ To examine the dose-response impact of the campaign, we aimed to air an additional 1600 TV GRPs in randomly selected local markets, representing an extra dose that was double that of the 2013 Tips national media buy, for a total of approximately 2400 GRPs.

The 20 largest US markets were excluded from randomisation eligibility because of the prohibitive expense of purchasing additional advertising in these markets. The remaining 190 markets were stratified by key characteristics associated with cigarette smoking prevalence (age, race/ethnicity, gender and education) and randomised within each stratum. Based on available funding for local media buys, the probability of random assignment to a higher dose within each stratum was 35\%. These parameters yielded 67 markets in the higher-dose group and 123 markets in the standard-dose group (figure 2).

To confirm similarity between the two randomised groups, we compared 2010 US Census demographic characteristics between the standard-dose and higher-dose markets. We also examined

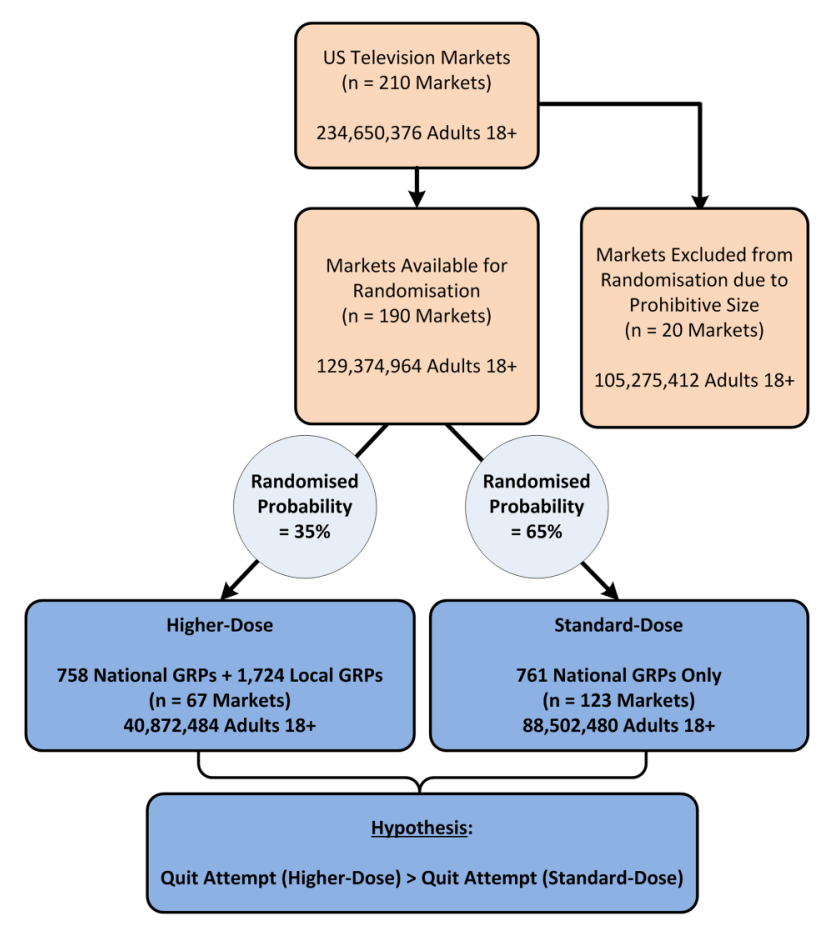

Figure 2 Flow diagram: randomised media dosing, 2013 Tips evaluation. GRP, gross rating point; Tips, Tips From Former Smokers. 
market-level cigarette smoking prevalence-aggregated across market counties and weighted by population-using 2012 Behavioral Risk Factor Surveillance System county-level smoking prevalence data. ${ }^{19}$ Finally, we examined differences in state-level tobacco control practices between the two randomised groups using recent state-level cigarette tax data ${ }^{20}$ and state per capita tobacco control expenditures. ${ }^{21}$

\section{Survey data and sample}

We analysed nationally representative survey data from smokers and non-smokers, respectively, collected shortly after the end of the 2013 Tips campaign (8 July 2013 to 1 October 2013). The sample was recruited from GfK's online KnowledgePanel $(\mathrm{KP})^{8} \quad 22 \quad 23$ and included all previously available and newly recruited smokers, as well as a random selection of nonsmokers. KP participants are recruited using address-based probability sampling, covering over $95 \%$ of US households. Because of the smaller number of higher-dose markets, panellists were oversampled from higher-dose markets to increase power. Panellists cannot volunteer for panel enrolment, and all participants have known probabilities of selection. Smokers were defined as adults aged 18 years or older who had smoked at least 100 cigarettes in their lifetime and reported currently smoking either every day or some days at the time of the 2013 Tips campaign launch. Non-smokers were adults aged 18 years or older who never smoked or did not smoke at the time of the survey. Current non-smokers who reported quitting since the launch of the campaign were considered to be smokers at the time of campaign launch and were counted as having made a quit attempt in the main smoker quit attempt analysis.

Survey weights were calculated to yield estimates representative of the markets in each randomised condition. A weighting procedure ${ }^{24}$ was performed to constrain each of the standarddose and higher-dose samples to a common distribution of age, gender, race/ethnicity and education matching the US Census distributions of these variables in the combined set of higherdose and standard-dose markets.

For smokers, the final analytic sample consisted of 3208 respondents in higher-dose markets and 2525 respondents in standard-dose markets. For non-smokers, the final analytic sample consisted of 1367 respondents in higher-dose markets and 1476 respondents in standard-dose markets. The overall survey completion rate was $56 \%$ among KP participants screened for survey participation. Power analysis was conducted based on anticipated effects of the higher-dose buy on quit attempts. Accounting for sample sizes in each randomised group, anticipated effect size of $4.4 \%$ based on limited data from a state campaign, ${ }^{7}$ market-level clustering and variability in market sample sizes, the sample was estimated to have $85 \%$ power to detect this size effect on the probability of a quit attempt.

\section{Measures}

Among smokers, the primary outcome was the incidence of making at least one quit attempt lasting 1 day or longer since the 2013 Tips campaign launch. Intentions to quit smoking (within 30 days and 6 months) were also measured as outcomes. Among non-smokers, key outcome measures were communication with friends or family about the dangers of smoking and encouragement to quit and use cessation resources. Among smokers and non-smokers, we also measured campaign awareness and exposure frequency. Dichotomous indicators assessed knowledge that health conditions highlighted in the 2013 Tips campaign-including tracheotomy, COPD, Buerger's disease, amputations, heart disease, stroke, diabetes complications and asthma - were related to smoking, as well as a non-related dummy condition (gallstones) and some smoking-related diseases not highlighted in 2013 ads.

Self-reported exposure to campaign advertisements was measured using a standard ad recognition protocol. ${ }^{25}$ Respondents viewed seven TV ads to prompt recall. All respondents viewed the six ads that were run most frequently, and a seventh ad chosen at random from the remaining five less-frequently shown ads. Immediately after viewing each ad, respondents were asked 'Have you seen this ad on television in the past (\# months) months since 4 March 2013?' Respondents could answer yes or no to this question. Respondents who answered yes to the ad recall question were then asked, 'In the past (\# months since 4 March 2013) months, how frequently have you seen this ad on television?' Response options included rarely, sometimes, often or very often. This process was repeated for each ad, with the ad display order randomised. Respondents unable to view ads via the within-survey video stream saw a screenshot storyboard and an ad script.

Overall campaign awareness was calculated as the percentage of the sample that indicated they had seen at least one 2013 Tips ad since the campaign launch. A frequency of exposure index was created to measure respondents' total exposure to these TV ads. This measure is defined as the sum of the mean recall frequencies $(0=$ never saw ad, $4=$ saw ad very often) across all 2013 Tips TV ads shown to the respondent.

Demographic covariates measured in the survey included age, gender, race/ethnicity, education, annual household income, TV hours per day, household presence of children aged 17 years or younger, household presence of a cigarette smoker, and having a chronic and/or mental health condition. In addition, external state-level and market-level variables were merged to the survey data, including cumulative state per capita tobacco control programme funding (1985-2012); state cigarette excise tax (2012); and market-level population size, median income (in tens of thousands of dollars) and the percentage of the population with a bachelor's degree. We also measured market-level cigarette smoking prevalence by aggregating recently published countylevel data on smoking prevalence ${ }^{19}$ to the market level, weighted by county population.

\section{Statistical analysis}

The impact of the higher dose was first assessed with weighted bivariate comparisons of key outcomes in the randomised higher-dose and standard-dose markets, with one-tailed statistical tests for between-market differences. We used one-tailed tests as our principal test of significance because our study was a real-world, pragmatic dosing test where all past research on cessation-focused campaigns, including the 2012 Tips evaluation, ${ }^{8}$ strongly suggested that increased dosing from a low baseline delivered over a relatively short time would either increase effects or have no effect. ${ }^{5} 711{ }^{12}{ }^{26-28}$ We then used multivariate analysis to estimate each outcome as a function of the higher-dose condition. We included in our multivariate models covariates for any individual, market or state-level characteristics that differed statistically between randomised dosing conditions. We also conducted additional stratified analyses among smokers to assess campaign effects by age, education, race/ethnicity, and presence of a chronic and/or mental health condition. The survey 'logistic' and 'regress' commands in Stata ${ }^{29}$ were used for model estimation, and estimated logistic coefficients were converted to odds ratios (ORs) to facilitate model interpretation. 


\section{RESULTS}

\section{Randomisation and sample characteristics}

The national media buy was almost identical between the standard-dose and higher-dose markets (761 vs 758 GRPs). The higher-dose markets received an additional 1724 GRPs. Age, gender, race/ethnicity and education distributions were identical between the standard-dose and higher dose markets after applying the weighting procedures. There was a small but statistically significant difference in market-level smoking prevalence between the higher-dose $(23.0 \%)$ and standard-dose markets (22.1\%). Mean state cigarette taxes, cumulative per capita state tobacco control programme expenditures and college completion were not significantly different between groups. There was a small but statistically significant difference in median income (US\$41.1 K higher-dose market; US\$42.5 K standard-dose market) and population size (944 K higher-dose market; $969 \mathrm{~K}$ standard-dose market).

Among smokers in the weighted survey sample (table 1), there were small but statistically significant differences in household income and presence of a mental health condition. The only significant difference by randomised higher-dose condition in the weighted non-smoker sample was presence of another smoker in the household. Covariates for these characteristics were included in our multivariate models of the associations between the higher-dose condition and each outcome variable for smokers and non-smokers.

\section{Ad exposure}

Among all respondents, $86.2 \%$ viewed the ads via in-survey video streaming; the remaining respondents referenced screenshots. Of the respondents viewing ads, $82.2 \%$ watched each ad for at least 20 seconds. Among smokers, $87.2 \%$ in higher-dose markets reported seeing at least one Tips ad compared with $75.0 \%$ in standard-dose markets $(\mathrm{p}<0.01)$ - an absolute difference of $12.2 \%$ (adjusted odds ratio $(\mathrm{aOR})=2.16 ; \mathrm{p}<0.01$ ), which represents a relative increase of $16.3 \%$ (table 2 ). The frequency of ad exposure index was higher among smokers in higher-dose markets than standard-dose markets (8.04 vs 5.09), a relative increase of $58.0 \%(\beta=2.85, \mathrm{p}<0.01$; table 2$)$. Average frequency of exposure overall was 8.06 for African-Americans versus 5.95 for Caucasians $(p<0.01)$. Frequency of exposure increased in higher-dose market from 6.86 to 10.56 among African-Americans $(\mathrm{p}<0.01)$ versus 4.99 to 7.95 among Caucasians $(\mathrm{p}<0.01)$.

\section{Impact on outcomes among all smokers}

Among smokers, the incidence of making a quit attempt was higher in higher-dose markets $(38.8 \%)$ than in standard-dose markets (34.9\%) —an absolute difference of 3.9\%, which represents a relative increase of $11.2 \%(\mathrm{aOR}=1.20 ; \mathrm{p}<0.03$; table 2$)$. Knowledge that amputations can result from smoking was higher $(\mathrm{p}<0.01)$ in higher-dose markets $(50.2 \%)$ than in standard-dose markets $(43.5 \%$; aOR $=1.31 ; \mathrm{p}<0.01)$, as was knowledge that smoking can worsen diabetic complications $(88.5 \%$ vs $84.2 \%$; $\mathrm{aOR}=1.43 ; \mathrm{p}<0.05)$ and knowledge of smoking-related COPD or chronic bronchitis $(91.8 \%$ vs $88.9 \%$; aOR $=1.35 ; \mathrm{p}<0.05)$. Intentions to quit smoking in the next 30 days or 6 months were not significantly different between higher-dose markets and standard-dose markets or for any of the remaining disease knowledge outcomes, although intention to quit in 6 months approached significance in the multivariate model $(p=0.06$; table 2).
Impact on ad exposure and outcomes among non-smokers

Among non-smokers, $83.9 \%$ of respondents in higher-dose markets reported seeing at least one Tips ad compared with $73.9 \%$ in standard-dose markets $(\mathrm{p}<0.01)$ —an absolute difference of $10.0 \%(\mathrm{aOR}=1.79 ; \mathrm{p}<0.01)$, which represents a relative increase of $13.5 \%$ (table 3 ). Frequency of exposure among non-smokers was $63.2 \%$ higher in higher-dose markets compared with standard-dose markets $(7.28$ vs $4.46, \mathrm{p}<0.01$; $\beta=2.61, p<0.01$ ).

Talking with friends or family about the dangers of smoking was more frequent among non-smokers $(p<0.01)$ in higherdose markets $(43.1 \%)$ than in standard-dose markets $(35.7 \%$; $\mathrm{aOR}=1.26, \mathrm{p}=0.03$; table 3 ). Similarly, encouraging others who smoke to quit was higher $(\mathrm{p}=0.03)$ among non-smokers in higher-dose markets $(45.5 \%)$ than in standard-dose markets $(40.0 \%)$, but this difference was not significant in multivariate analysis $(\mathrm{aOR}=1.14, \mathrm{p}=0.14)$. Knowledge of smoking-related diseases was significantly greater in higher-dose markets than in standard-dose markets for lung cancer $(\mathrm{aOR}=2.64)$, cancer of the mouth or throat $(\mathrm{aOR}=2.65)$, heart disease $(\mathrm{aOR}=1.65)$, emphysema $(\mathrm{aOR}=1.65)$, hole in throat (stoma or tracheotomy; $\mathrm{aOR}=2.19)$, amputations $(\mathrm{aOR}=1.67)$, asthma $(\mathrm{aOR}=1.59)$, COPD (or chronic bronchitis; $\mathrm{aOR}=2.48$ ), and worsening of diabetic complications $(\mathrm{aOR}=1.70)$ in bivariate and multivariate analysis (all p $<0.05)$. Knowledge of stroke as a smoking-related disease was significantly higher in bivariate analysis $(\mathrm{p}<0.01)$, and at the significance margin in multivariate analysis $(\mathrm{aOR}=1.34, \mathrm{p}=0.05)$.

\section{Impact on quit attempts among smokers by demographic subgroup}

The increase in quit attempt incidence in higher-dose markets compared with standard-dose markets was greater for some demographic subgroups (table 4). African-American smokers reported markedly higher quit attempt incidence in higher-dose markets $(50.9 \%)$ compared with standard-dose markets $(31.8 \%$; $\mathrm{aOR}=1.96 ; \mathrm{p}<0.01)$. Quit attempt rates were higher $(\mathrm{p}<0.05)$ among Hispanic smokers $(56.3 \%)$ in higher-dose markets than in standard-dose markets (39.9\%); however, this difference was not significant in multivariate analysis $(\mathrm{aOR}=1.30 ; \mathrm{p}=0.27)$. There was no statistically significant difference $(p=0.23)$ in the quit attempt rate among Caucasians between higher-dose (33.4\%) and standard-dose (34.8\%) markets. Smokers with only some college education reported higher quit attempt incidence in higher-dose markets (45.4\%) compared with standard-dose markets $(33.9 \%$; $a \mathrm{OR}=1.60 ; \mathrm{p}<0.01)$. However, there was no significant effect in smokers who completed college. Respondents with chronic diseases (non-mental) reported higher quit attempt rates in higher-dose markets (39.3\%) compared with standard-dose markets $(32.0 \%$; $\mathrm{aOR}=1.21$; $\mathrm{p}<0.03)$. Respondents without a mental health condition reported higher quit attempt rates in higher-dose markets than standard-dose markets $(38.5 \%$ vs $32.0 \%$; p < 0.01$)$, whereas respondents with a mental health condition did not $(39.5 \%$ vs $42.5 \% ; \mathrm{p}=0.79)$. However, among respondents in standarddose markets, the quit attempt rate was higher among those reporting a mental health condition than among those not reporting a mental health condition $(42.5 \%$ vs $32.0 \%$; $<<0.01)$.

\section{DISCUSSION}

Higher-dose local ad buys in randomly selected markets in the 2013 Tips study generated a 3.9\% absolute increase $(11.2 \%$ relative increase) in quit attempts above the base rate in 


\begin{tabular}{|c|c|c|c|c|c|c|c|c|}
\hline \multirow[b]{3}{*}{ Characteristic } & \multicolumn{4}{|l|}{ Cigarette smokers } & \multicolumn{4}{|l|}{ Non-smokers } \\
\hline & \multicolumn{2}{|l|}{ Unweighted (\%)* } & \multicolumn{2}{|l|}{ Weighted (\%)† } & \multicolumn{2}{|l|}{ Unweighted (\%)* } & \multicolumn{2}{|l|}{ Weighted (\%)† } \\
\hline & $\begin{array}{l}\text { Standard-dose markets } \\
(\mathrm{n}=2525)\end{array}$ & $\begin{array}{l}\text { Higher-dose markets } \\
(\mathrm{n}=3208)\end{array}$ & $\begin{array}{l}\text { Standard-dose markets } \\
(\mathrm{n}=2525)\end{array}$ & $\begin{array}{l}\text { Higher-dose markets } \\
(\mathrm{n}=3208)\end{array}$ & $\begin{array}{l}\text { Standard-dose markets } \\
(\mathrm{n}=1476)\end{array}$ & $\begin{array}{l}\text { Higher-dose markets } \\
(n=1367)\end{array}$ & $\begin{array}{l}\text { Standard-dose markets } \\
(\mathrm{n}=1476)\end{array}$ & $\begin{array}{l}\text { Higher-dose } \\
\text { markets } \\
(n=1367)\end{array}$ \\
\hline \multicolumn{9}{|l|}{ Age } \\
\hline $18-24$ & 5.5 & 3.8 & 13.5 & 13.5 & 7.6 & 4.7 & 13.5 & 13.5 \\
\hline $25-34$ & 16.4 & 10.3 & 17.0 & 17.0 & 15.1 & 12.4 & 17.0 & 17.0 \\
\hline $35-54$ & 40.6 & 43.1 & 35.7 & 35.7 & 33.8 & 31.7 & 35.7 & 35.7 \\
\hline $55+$ & 37.5 & $42.8 \S$ & 33.8 & 33.8 & 43.5 & $51.2 \S$ & 33.8 & 33.8 \\
\hline \multicolumn{9}{|l|}{ Gender } \\
\hline Male & 36.2 & 30.6 & 48.7 & 48.7 & 40.9 & 36.9 & 48.7 & 48.7 \\
\hline Female & 63.8 & $69.4 \S$ & 51.3 & 51.3 & 59.1 & $63.1 \ddagger$ & 51.3 & 51.3 \\
\hline \multicolumn{9}{|l|}{ Race/ethnicity } \\
\hline White, non-Hispanic & 79.1 & 84.9 & 74.1 & 74.1 & 80.0 & 82.9 & 74.1 & 74.1 \\
\hline Black, non-Hispanic & 6.8 & 5.1 & 11.0 & 11.0 & 5.3 & 5.9 & 11.0 & 11.0 \\
\hline Hispanic & 7.9 & 4.9 & 9.9 & 9.9 & 9.1 & 6.0 & 9.9 & 9.9 \\
\hline Other, non-Hispanic & 6.1 & $5.1 \S$ & 4.9 & 4.9 & 5.6 & $5.2 \ddagger$ & 4.9 & 4.9 \\
\hline \multicolumn{9}{|l|}{ Educational attainment } \\
\hline Less than high school & 7.5 & 6.5 & 14.8 & 14.8 & 3.7 & 5.0 & 14.8 & 14.8 \\
\hline High school graduate & 26.2 & 25.8 & 31.1 & 31.1 & 21.8 & 19.2 & 31.1 & 31.1 \\
\hline Some college & 47.2 & 47.5 & 31.6 & 31.6 & 33.7 & 36.2 & 31.6 & 31.6 \\
\hline College graduate or more & 19.2 & 20.2 & 22.5 & 22.5 & 40.9 & 39.7 & 22.5 & 22.5 \\
\hline \multicolumn{9}{|l|}{ Annual household income } \\
\hline Less than $\$ 20000$ & 25.0 & 22.8 & 23.6 & 23.2 & 12.7 & 12.6 & 14.7 & 15.1 \\
\hline$\$ 20000-\$ 49999$ & 37.4 & 37.8 & 31.2 & 31.6 & 30.5 & 31.2 & 28.2 & 29.3 \\
\hline$\$ 50000-\$ 99999$ & 30.2 & 30.5 & 36.1 & 32.2 & 37.0 & 38.3 & 38.0 & 38.4 \\
\hline$\$ 100000$ or more & 7.4 & 8.9 & 9.1 & $12.9 \ddagger$ & 19.8 & 18.0 & 19.0 & 17.2 \\
\hline \multicolumn{9}{|l|}{ TV hours per day } \\
\hline 1 or more hours & 89.1 & 89.6 & 87.3 & 87.4 & 85.3 & $90.6 \S$ & 86.9 & 88.9 \\
\hline \multicolumn{9}{|l|}{ Children in the household } \\
\hline 1 or more & 33.6 & $31.0 \ddagger$ & 37.4 & 36.9 & 31.2 & 28.1 & 35.8 & 37.2 \\
\hline \multicolumn{9}{|c|}{ Has a chronic condition, non-mental } \\
\hline Yes & 71.5 & $79.5 \S$ & 67.1 & 71.2 & 70.3 & $79.0 \S$ & 63.4 & 69.3 \\
\hline \multicolumn{9}{|c|}{ Has a mental health condition } \\
\hline Yes & 32.1 & $34.7 \ddagger$ & 28.2 & $32.2 \ddagger$ & 20.7 & 22.2 & 17.7 & 22.2 \\
\hline \multicolumn{9}{|c|}{ Another smoker in the household } \\
\hline Yes & 44.8 & 46.4 & 46.2 & 49.0 & 10.4 & $16.6 \S$ & 10.8 & $15.7 \ddagger$ \\
\hline
\end{tabular}


Table 2 Randomised media higher-dose results among cigarette smokers, ${ }^{*} 2013$ Tips evaluation

\begin{tabular}{|c|c|c|c|c|c|}
\hline \multirow[b]{2}{*}{ Smoker outcome variables } & \multicolumn{3}{|l|}{ Descriptive statistics } & \multicolumn{2}{|l|}{ Multivariate modelst } \\
\hline & Standard-dose markets & Higher-dose markets & p Value $\ddagger$ & Higher-dose adjusted OR & p Value $\ddagger$ \\
\hline \multicolumn{6}{|l|}{ Ad exposure } \\
\hline Awareness of any Tips ad on TV & $75.0 \S$ & 87.2 & $<0.001$ & 2.16 & $<0.001$ \\
\hline Frequency of exposure to Tips ads & 5.09 & 8.04 & $<0.001$ & $2.85^{* *}$ & $<0.001$ \\
\hline \multicolumn{6}{|l|}{ Quit attempts and intentions to quit } \\
\hline Incidence of quit attempt since 2013 Tips launch & 34.9 & 38.8 & 0.039 & 1.20 & 0.029 \\
\hline Intends to quit in next 30 dayst† & 12.2 & 13.4 & 0.235 & 1.16 & 0.131 \\
\hline Intends to quit in next 6 monthst† & 23.5 & 25.8 & 0.114 & 1.16 & 0.059 \\
\hline \multicolumn{6}{|l|}{ Disease knowledge (campaign-related) } \\
\hline Heart disease & 83.5 & 84.9 & 0.219 & 1.09 & 0.255 \\
\hline Stroke & 74.7 & 77.7 & 0.066 & 1.14 & 0.114 \\
\hline Hole in throat (stoma or tracheotomy) & 83.3 & 83.6 & 0.412 & 1.05 & 0.314 \\
\hline Buerger's disease & 28.6 & 31.7 & 0.077 & 1.12 & 0.135 \\
\hline Amputations & 43.5 & 50.2 & 0.001 & 1.31 & 0.002 \\
\hline Asthma & 79.5 & 81.7 & 0.104 & 1.17 & 0.058 \\
\hline COPD or chronic bronchitis & 88.9 & 91.8 & 0.025 & 1.35 & 0.038 \\
\hline Worsening of diabetic complications & 84.2 & 88.5 & 0.003 & 1.43 & 0.010 \\
\hline \multicolumn{6}{|l|}{ Disease knowledge (unrelated to campaign) } \\
\hline Lung cancer & 94.3 & 95.4 & 0.129 & 1.24 & 0.118 \\
\hline Cancer of mouth or throat & 90.8 & 91.8 & 0.215 & 1.15 & 0.182 \\
\hline Diabetes & 26.9 & 27.6 & 0.360 & 1.01 & 0.455 \\
\hline Emphysema & 92.9 & 92.3 & 0.673 & 0.91 & 0.674 \\
\hline Gallstones (control item) & 17.0 & 16.5 & 0.598 & 0.95 & 0.649 \\
\hline
\end{tabular}

*Among current smokers at time of 2013 Tips launch.

†Multivariate models control for income, mental health condition, media market population size, media market smoking prevalence and median income in media market. Survey weights control for differences in age, gender, race/ethnicity and educational attainment across randomised markets.

$\ddagger p$ Values are calculated as one-tailed (higher-dose greater than standard-dose); $p<0.05$.

§Statistically significant differences are indicated in boldface type.

१Frequency is expressed as an integer.

** Ordinary least squares $\beta$ coefficient.

t†Among current smokers at time of survey only.

COPD, chronic obstructive pulmonary disease; Tips, Tips From Former Smokers; TV, television.

standard-dose markets (34.9\% vs $38.8 \%)$. Consistent with the results of a 2012 longitudinal cohort trial, these findings from a randomisation of markets in 2013 indicate the effectiveness of media campaigns for rapidly increasing quit attempts among smokers and engaging non-smokers. The 2012 Tips evaluation used a pre-post analysis that determined the campaign generated a $3.7 \%$ absolute increase in quit attempts nationally from $31.1 \%$ to $34.8 \%$, resulting in 1.6 million quit attempts. ${ }^{8}$

The 2013 higher-dose ad buy had a more substantial impact on African-Americans, who had a 50.9\% quit attempt rate in higherdose markets compared with $31.8 \%$ in standard-dose markets (60.1\% relative increase). This dramatic relative increase may be related to increased ad exposure and receptivity, as documented in previous studies examining African-American antismoking ad response. For example, some prior studies have shown that African-Americans are more likely to rate antismoking ads high on measures of 'perceived ad effectiveness', which is predictive of smoking-related outcomes. ${ }^{30} 31$ Additionally, bivariate analysis revealed substantial increases in quit attempts among Hispanics (56.3\% vs 39.9\%), although this increase was not statistically significant by multivariate analysis. This lack of significance despite a 1.3 OR could be related to insufficient power to detect a subpopulation effect. We also observed an increased impact among those completing only some college without graduating, which represents almost a third of US adults. ${ }^{32}$ Those with chronic physical conditions benefitted from the higher-dose buy, which may reflect pre-existing increased motivation because of the effects of smoking. The standard-dose quit attempt rate was higher in those with mental health conditions than those without; however, no additional dose effect was observed. This is consistent with other recent evidence suggesting that smokers with mental illness are more likely to make quit attempts than those without mental illness, although their success rate is lower. ${ }^{33}$

Among smokers and non-smokers, knowledge of smoking-related disease conditions was generally greater in higher-dose markets. This impact was stronger for the disease conditions explicitly highlighted in the 2013 Tips campaign. No significant impact was observed for conditions unrelated to the 2013 Tips ads, with the exception of lung cancer and oral cancer in non-smokers (emphysema, which is a form of COPD, was significant in multivariate but not bivariate analysis). The lung cancer and oral cancer exceptions may have been because of a tracheostomy ad and a lung surgery ad (for COPD) that viewers interpreted as related to oral and lung cancer. In general, our results demonstrate that the campaign affected targeted outcomes and not unrelated ones. For example, knowledge that smoking worsens diabetic complications was higher with the higher dose, whereas knowledge that smoking causes diabetes was not. This is consistent with the focus of the 2013 diabetes ad, which focused on worsening complications in a person with existing diabetes.

Among non-smokers, talking with friends or family about the dangers of smoking was significantly more frequent in higherdose markets. Increases in campaign-related disease knowledge among non-smokers were even more consistently associated with campaign ads than among smokers. These findings may be 
Table 3 Randomised media higher-dose results among non-smokers, 2013 Tips evaluation

\begin{tabular}{|c|c|c|c|c|c|}
\hline \multirow[b]{2}{*}{ Non-smoker outcome variables } & \multicolumn{3}{|c|}{ Descriptive statistics } & \multicolumn{2}{|c|}{ Multivariate models* } \\
\hline & $\begin{array}{l}\text { Standard-dose } \\
\text { markets }\end{array}$ & $\begin{array}{l}\text { Higher-dose } \\
\text { markets }\end{array}$ & p Valuet & $\begin{array}{l}\text { Higher-dose } \\
\text { adjusted OR }\end{array}$ & p Valuet \\
\hline \multicolumn{6}{|l|}{ Ad exposure } \\
\hline Awareness of any Tips ad on TV & $73.9 \ddagger$ & 83.9 & $<0.001$ & 1.79 & $<0.001$ \\
\hline Frequency of exposure to Tips ads§ & 4.46 & 7.28 & $<0.001$ & 2.619 & $<0.001$ \\
\hline \multicolumn{6}{|l|}{ Non-smokers' communication with others } \\
\hline Talked to friends/family about dangers of smoking & 35.7 & 43.1 & 0.005 & 1.26 & 0.029 \\
\hline Encouraged friend/family who smoke to quit & 40.0 & 45.5 & 0.033 & 1.14 & 0.135 \\
\hline Recommended friend/family who smoke to use cessation resources & 3.4 & 4.1 & 0.271 & 1.07 & 0.411 \\
\hline \multicolumn{6}{|l|}{ Disease knowledge (campaign related) } \\
\hline Heart disease & 86.8 & 91.0 & 0.017 & 1.65 & 0.012 \\
\hline Stroke & 80.4 & 84.6 & 0.029 & 1.34 & 0.052 \\
\hline Hole in throat (stoma or tracheotomy) & 85.5 & 92.0 & $<0.001$ & 2.19 & $<0.001$ \\
\hline Buerger's disease & 33.8 & 37.3 & 0.127 & 1.20 & 0.088 \\
\hline Amputations & 45.6 & 57.6 & $<0.001$ & 1.67 & $<0.001$ \\
\hline Asthma & 84.7 & 89.7 & 0.004 & 1.59 & 0.002 \\
\hline COPD or chronic bronchitis & 88.9 & 95.4 & $<0.001$ & 2.48 & $<0.001$ \\
\hline Worsening of diabetic complications & 92.1 & 94.9 & 0.034 & 1.70 & 0.134 \\
\hline \multicolumn{6}{|l|}{ Disease knowledge (unrelated to campaign) } \\
\hline Lung cancer & 95.5 & 98.1 & 0.004 & 2.64 & 0.002 \\
\hline Cancer of mouth or throat & 93.6 & 97.2 & $<0.001$ & 2.65 & $<0.001$ \\
\hline Diabetes & 31.2 & 33.8 & 0.185 & 1.15 & 0.157 \\
\hline Emphysema & 91.1 & 93.9 & 0.069 & 1.65 & 0.046 \\
\hline Gallstones (control item) & 23.2 & 22.5 & 0.596 & 0.98 & 0.547 \\
\hline
\end{tabular}

of particular interest in countries where fewer tobacco control policies are in place, public awareness of the dangers of smoking is low or the baseline quit attempt rate is low. In this circumstance, higher-dose media campaigns might increase nonsmoker support for policy change and increase friends or family talking about the dangers of smoking. However, further country-specific consideration and evaluation of the relative costs and benefits is warranted.

The additional dose to create the overall incremental effects in 2013 was significantly larger (approximately 1700 GRPs) than the national dose delivered in 2012 (1000 GRPs). Consequently, the 2012 Tips campaign required about 270 GRPs per percentage point increase in overall quit attempts, whereas 2013 Tips higher dose required over 400 additional GRPs per percentage point increase. This comparison is based on the presumptive equivalence in execution between the 2012 and 2013 campaigns, because each campaign used similar message styles, themes and even some of the same ad participants. However, a higher-dose strategy may be efficient for subgroups where larger effects were seen, most notably for African-American smokers, who had a 19.1\% absolute increase. Because higher-dose local buys are often more expensive, purchasing more national coverage, including via African-American national media channels, may be more costeffective. A return-on-investment study is in progress to examine this and other questions.

Randomisation of a mass media intervention at the level of delivery in a majority of US markets is a strength of this study because randomisation decreases the likelihood of confounding or reverse causation accounting for detected effects. Measurement of quit attempts (an important public health outcome), large sample size, multivariate analysis to account for variation between randomised markets, examination of effects on non-smokers and key subpopulations of smokers, and use of address-based nationally representative samples are also strengths.

\section{Limitations and applicability}

Our study has several limitations. Although we used a probability sample designed to be representative of the higher-dose market and standard-dose market populations, the surveys were conducted online, and respondents who joined the study may have been different from those who declined. In addition, Tips campaigns have been relatively short (3-4 months) compared with other regional and country-level campaigns. Also, although having survey follow-up occur immediately after the campaign may be a strength for detecting immediate effects, we were unable to measure delayed effects on quit attempts and longterm abstinence beyond the follow-up window. In addition, we did not have sufficient demographic information or statistical power to explore differential effects on other key subpopulations, such as sexual and gender minorities and other racial/ ethnic groups. Further, any lack of positive findings for subpopulations we did examine should be interpreted with caution because sample size varied considerably. For example, the lack of effect for Hispanics with multivariate analysis despite a large absolute and relative percentage increase may be due to a lack of statistical power. 
Table 4 Quit attempt incidence by demographics and medical conditions among cigarette smokers, ${ }^{*} 2013$ Tips evaluation

\begin{tabular}{|c|c|c|c|c|c|}
\hline \multirow[b]{2}{*}{ Characteristic } & \multicolumn{3}{|c|}{ Descriptive statistics (quit attempt prevalence) } & \multicolumn{2}{|l|}{ Multivariate models $t$} \\
\hline & Standard-dose markets (\%) & Higher-dose markets (\%) & p Value $\neq$ & Higher-dose adjusted OR & p Value $\ddagger$ \\
\hline \multicolumn{6}{|l|}{ Age (years) } \\
\hline $18-44$ & 39.4 & 44.3 & 0.106 & 1.27 & 0.075 \\
\hline $45+$ & 30.9 & 34.2 & 0.061 & $1.18 \S$ & 0.048 \\
\hline \multicolumn{6}{|l|}{ Education } \\
\hline High school graduate or less & 32.9 & 32.4 & 0.560 & 0.96 & 0.613 \\
\hline Less than high school & 33.9 & 36.0 & 0.394 & 1.19 & 0.307 \\
\hline High school graduate only & 32.4 & 30.6 & 0.686 & 0.86 & 0.818 \\
\hline Some college & 33.9 & 45.4 & $<0.001$ & 1.60 & $<0.001$ \\
\hline College graduate & 40.6 & 42.6 & 0.328 & 1.14 & 0.241 \\
\hline \multicolumn{6}{|l|}{ Race/ethnicity } \\
\hline White & 34.8 & 33.4 & 0.732 & 0.95 & 0.245 \\
\hline African-American & 31.8 & 50.9 & 0.006 & 1.96 & 0.005 \\
\hline Hispanic & 39.9 & 56.3 & 0.043 & 1.30 & 0.268 \\
\hline \multicolumn{6}{|l|}{ Chronic condition } \\
\hline Has a chronic (non-mental) condition & 32.0 & 39.3 & 0.007 & 1.21 & 0.028 \\
\hline Does not have chronic (non-mental) condition & 33.6 & 36.4 & 0.281 & 1.23 & 0.161 \\
\hline Has a mental health condition & 42.5 & 39.5 & 0.797 & 0.93 & 0.689 \\
\hline Does not have a mental health condition & 32.0 & 38.5 & 0.008 & 1.37 & 0.003 \\
\hline
\end{tabular}

${ }^{*}$ Among current smokers at time of 2013 Tips launch.

tMultivariate models control for income, mental health condition, media market population size, media market smoking prevalence and median income in media market. Survey

weights control for differences in age, gender, race/ethnicity and educational attainment across randomised markets.

$\neq p$ Values are calculated as one-tailed (higher-dose greater than standard-dose), $p<0.05$.

$\S$ Statistically significant differences are indicated in boldface type.

Tips, Tips From Former Smokers.

Application and interpretation of our results may be influenced by how media markets function, how we measured recall and the baseline dose in the standard condition. Regional versus national buy efficiency may depend on local specifics of how media markets are segmented and purchased and how subpopulations react to media campaigns. We measured recall of any ad based on showing each respondent 7 of 11 ads that were played during the campaign. Consequently, our reported recall fraction may be lower than would have been the case if we had shown respondents all 11 ads. In addition, a lack of positive findings for comparisons made in this study is only relevant to the question of incremental impact of a higher dose above baseline. Because all respondents received a baseline dose, a lack of positive findings from the higher dose should not be interpreted to mean that baseline media does not produce an effect.

Because the 20 largest US markets were excluded due to prohibitive costs, our findings reflect the impact of additional media on small-sized to medium-sized markets. Furthermore, the tobacco control policy environment in the 20 largest markets is generally more favourable than that of the study markets (eg, 85.9\% clean indoor air law vs 68.2\%; state excise tax $\$ 1.61$ vs $\$ 1.29$ ). Prior evaluation of mass media campaigns suggests they produce larger effects when conducted in markets with complementary tobacco control policies. ${ }^{10}$ Consequently, our findings may underestimate the effect size of a higher dose at the national level that would include large markets. Finally, we were unable to adjust for heavier smoking because this was a postintervention-only survey, and we cannot accurately establish the respondents' dependence status prior to the campaign without assuming that dependence level did not change during the campaign time period.

\section{CONCLUSIONS}

Future research and economic modelling ${ }^{34}$ may be useful to further hone the application of these findings to future campaigns. Because we focused solely on cigarettes, evaluation of future campaigns that include other tobacco products may be useful. Campaign developers and evaluators may benefit from examining differential campaign effects on key subpopulations. Development and evaluation of future ads may benefit from additional exploration of the impact of ads on groups responding less strongly, such as those with no college education, Caucasians and those with a mental health condition.

In 2014, the US Surgeon General released the 50th anniversary report on the health consequences of smoking. Although 8 million lives had been saved by tobacco control nationally, 5.6 million US children alive today will die prematurely from smoking if more dramatic action is not taken. One billion people worldwide will die in the 21st century unless trends are reversed. One of the report's key recommendations was to counteract "industry marketing by sustaining high impact national media campaigns like the CDC's Tips From Former Smokers campaign and FDA's youth prevention campaigns at a high frequency level and exposure for 12 months a year for a decade or more (p. 875)." 2 We now have conducted two rigorous country-level real-world trials: a longitudinal pre-post cohort study in $2012^{8}$ and a randomised field trial of market-level dosing in 2013. Both studies demonstrate the effectiveness of carefully designed and delivered media campaigns for engaging non-smokers and rapidly increasing quit attempts among smokers, especially African-Americans, those with only some college education, and those with a non-mental health chronic disease. Our findings add a new level of empirical support to previous studies conducted in low-income and middle-income countries ${ }^{35}$ and consequently support WHO's MPOWER recommendation to all countries for investment in tobacco education. ${ }^{3}$ 


\section{What this paper adds}

- Tobacco control strategies, including public education campaigns, have been shown to be effective in promoting behavioural changes, including incidence of quit attempts.

- The 2012 US Tips From Former Smokers national tobacco education campaign generated an estimated 1.6 million population-level quit attempts.

- Evaluation of the impact of higher doses of tobacco prevention media campaigns has generally been limited to state-based or regional campaigns using non-randomised designs.

- Little is known about the effects of media dosing above Centers for Disease Control and Prevention (CDC)-recommended minimum advertising levels on cessation behaviour.

- Examination of campaign effects on key subpopulationssuch as lower education, racial/ethnic minorities, and those with chronic physical and mental health conditions-has been lacking.

- Our findings demonstrate the effectiveness of higher doses of media on rapidly increasing quit attempts among smokers, especially among African-Americans.

- Non-smokers report increased conversations with family or friends about the dangers of smoking and have greater knowledge of smoking-related diseases at a higher media dose.

- This is the first study to demonstrate the effectiveness of a national tobacco education campaign using field-based randomisation at the media market level.

Acknowledgements The authors thank the 11 former smokers who shared their personal stories with the public in the 2013 Tips campaign, especially recognising the contribution of Nathan Moose, Bill Busse and Terrie Hall, who passed away from their smoking-related illnesses after the campaign. They also thank Diane Beistle and Jane Mitchko who oversaw the Tips campaign, and Robert Rodes, who oversaw execution of the randomised higher dose media buy. They also thank Terry F Pechacek, Xin Xu, Ralph Caraballo, Jami Fraze, Ann Malarcher, Steve Babb, Shanna Cox, Michelle Johns, Gabbi Promoff and Brian King for scientific, programmatic and technical support; staff of CDC's Office on Smoking and Health; Ursula Bauer and Thomas Frieden for mobilising CDC support and providing scientific support; the Plowshare Group for undertaking the media campaign; and Jonathan Blitstein, Jennifer Duke, and Burton Levine of RTI International for data analysis and technical support.

Contributors All authors were involved in study design, analysis of the data, development of figures, interpretation, and writing of the report. The corresponding author had full access to all data in the study and had final responsibility for the decision to submit for publication.

Funding US Department of Health and Human Services, Centers for Disease Control and Prevention.

Disclaimer The findings and conclusions in this report are those of authors and do not necessarily represent the official position of the Centers for Disease Control and Prevention or RTI International.

Ethics approval This study was approved by the Institutional Review Board of RTI International.

Provenance and peer review Not commissioned; externally peer reviewed.

Data sharing statement All television, radio and print ads are available online at http://www.cdc.gov/tips.

Open Access This is an Open Access article distributed in accordance with the Creative Commons Attribution Non Commercial (CC BY-NC 4.0) license, which permits others to distribute, remix, adapt, build upon this work non-commercially, and license their derivative works on different terms, provided the original work is properly cited and the use is non-commercial. See: http://creativecommons.org/ licenses/by-nc/4.0/

\section{REFERENCES}

1 World Health Organization. WHO global report: mortality attributable to tobacco. Geneva, Switzerland: World Health Organization, 2012.

2 US Department of Health and Human Services (USDHHS). The health consequences of smoking - 50 years of progress: a report of the Surgeon General. Atlanta, GA: US Department of Health and Human Services, Centers for Disease Control and Prevention, National Center for Chronic Disease Prevention and Health Promotion, Office on Smoking and Health, 2014.

3 World Health Organization. MPOWER in action. Geneva, Switzerland: World Health Organization, 2013.

4 Centers for Disease Control and Prevention. Best practices for comprehensive tobacco control programs-2014. Atlanta: U.S. Department of Health and Human Services, Centers for Disease Control and Prevention, National Center for Chronic Disease Prevention and Health Promotion, Office on Smoking and Health, 2014.

5 Davis KC, Nonnemaker JM, Farrelly MC. Association between national smoking prevention campaigns and perceived smoking prevalence among youth in the United States. J Adolesc Health 2007:41:430-6.

6 Durkin S, Brennan E, Wakefield M. Mass media campaigns to promote smoking cessation among adults: an integrative review. Tob Control 2012;21:127-38.

7 Farrelly MC, Duke JC, Davis KC, et al. Promotion of smoking cessation with emotional and/or graphic antismoking advertising. Am J Prev Med 2012;43:475-82

8 McAfee T, Davis KC, Alexander RL Jr, et al. Effect of the first federally funded US antismoking national media campaign. Lancet 2013;382:2003-11.

9 Murukutla N, Turk T, Prasad CV, et al. Results of a national mass media campaign in India to warn against the dangers of smokeless tobacco consumption. Tob Control 2012;21:12-17.

10 National Cancer Institute. The role of the media in promoting and reducing tobacco use: tobacco control monograph no. Bethesda, MD: US. Department of Health and Human Services, National Institutes of Health, National Cancer Institute, 2008.

11 Sims M, Salway R, Langley T, et al. Effectiveness of tobacco control television advertising in changing tobacco use in England: a population-based cross-sectional study. Addiction 2014;109:986-94.

12 Vallone DM, Duke JC, Cullen J, et al. Evaluation of EX: a national mass media smoking cessation campaign. Am J Public Health 2011;101:302-9.

13 Wakefield MA, Spittal MJ, Yong $\mathrm{HH}$, et al. Effects of mass media campaign exposure intensity and durability on quit attempts in a population-based cohort study. Health Educ Res 2011;26:988-97.

14 Duke JC, Davis KC, Alexander RL, et al. Impact of a U.S. antismoking national media campaign on beliefs, cognitions and quit intentions. Health Educ Res 2015;30:466-83.

15 Centers for Disease Control and Prevention. Tips from former smokers. 2012. http:/l www.cdc.gov/tobacco/campaign/tips/ (accessed 5 Dec 2014).

16 Davis KC, Farrelly MC, Messeri P, et al. The impact of national smoking prevention campaigns on tobacco-related beliefs, intentions to smoke and smoking initiation: results from a longitudinal survey of youth in the United States. Int I Environ Res Public Health 2009;6:722-40.

17 Duke JC, Vallone DM, Allen J, et al. Increasing youths' exposure to a tobacco prevention media campaign in rural and low population density communities. Am J Public Health 2009:99:2210-16.

18 Centers for Disease Control and Prevention. Best practices for comprehensive tobacco control programs-2007. Atlanta, GA: Department of Health and Human Services, 2007.

19 Dwyer-Lindgren L, Mokdad AH, Srebotnjak T, et al. Cigarette smoking prevalence in US counties: 1996-2012. Popul Health Metr 2014;12:5.

20 Orzechowski \& Walker Consulting Firm. The tax burden on tobacco. Vol 47 Arlington, VA, 2012

21 Farrelly MC, Pechacek TF, Chaloupka FJ. The impact of tobacco control program expenditures on aggregate cigarette sales: 1981-2000. J Health Econ 2003;22:843-59

22 Chang L, Krosnick JA. National surveys via RDD telephone interviewing versus the Internet. Public Opin Q 2009;73:641-78.

23 Yeager DS, Krosnick JA, Chang L, et al. Comparing the accuracy of RDD telephone surveys and Internet surveys conducted with probability and non-probability samples. Public Opin Q 2011;75:709-47.

24 Centers for Disease Control and Prevention. Methodologic changes in the Behavioral Risk Factor Surveillance System in 2011 and potential effects on prevalence estimates. MMWR Morb Mortal Wkly Rep 2012;61:410-13.

25 Southwell BG, Barmada CH, Hornik RC, et al. Can we measure encoded exposure? Validation evidence from a national campaign. J Health Commun 2002;7:445-53.

26 Durkin SJ, Biener L, Wakefield MA. Effects of different types of antismoking ads on reducing disparities in smoking cessation among socioeconomic subgroups. Am J Public Health 2009;99:2217-23.

27 Cho H, Abe S. Is two-tailed testing for directional research hypotheses legitimate? J Bus Res 2013:66:1261-6.

28 Ludbrook J. Should we use on-sided or two-sided P values in tests of significance? Clin Exp Pharmacol Physio 2013;40:357-61. 
29 StataCorp. Stata: release 13. Statistical software: StataCorp LP, 2013.

30 Davis KC, Nonnemaker J, Duke J, et al. Perceived effectiveness of cessation advertisements: The importance of audience reactions and practical implications for media campaign planning. Health Commun 2013; 28:461-72.

31 Davis KC, Nonnemaker JM, Farrelly MC, et al. Exploring differences in smokers' perceptions of the effectiveness of cessation media messages. Tob Control 2011;20:26-33.

32 U.S. Census Bureau. Table 1. Educational attainment of the population 18 years and over, by age, sex, race, and Hispanic origin: 2013. Current Population Survey:
Annual Social and Economic Supplement. https://www.census.gov/hhes/socdemo/ education/data/cps/2013/tables.html (accessed 13 Nov 2015).

33 Morris CD, Burns EK, Waxmonsky JA, et al. Smoking cessation behaviors among persons with psychiatric diagnoses: results from a population-level state survey. Drug Alcohol Depend 2014;136:63-8.

$34 \mathrm{Xu} \mathrm{X}$, Alexander RL Jr, Simpson SA, et al. A cost-effectiveness analysis of the first federally funded antismoking campaign. Am J Prev Med 2015;48:318-25.

35 Wakefield M, Bayly M, Durkin S, et al. Smokers' responses to television advertisements about the serious harms of tobacco use: pre-testing results from 10 low- to middle-income countries. Tob Control 2013;22:24-31. 